\title{
REFERENCES
}

1. Marilyn Breen, Intersecting unions of maximal convex sets, Proc. Amer. Math. Soc. 39 (1973), 587-590. MR 47 \#7592.

2. Weir Katchalski, The dimension of intersections of convex sets, Israel J. Math. 10 (1971), 465-470. MR $46 \# 4367$.

DEP ARTMENT OF MATHEMATICS, UNIVERSITY OF OKLAHOMA, NORMAN, OKLAHOM A 73069

PROCEEDINGS OF THE

AMERICAN MATHEMATICAL SOCIETY

Volume 51, Number 2, September 1975

\section{A CHARACTERIZATION OF THE KERNEL OF A CLOSED SET}

\author{
MARILYN BREEN
}

ABSTRACT. Let $S$ be a closed subset of some linear topological space such that int ker $S \neq \varnothing$ and $\operatorname{ker} S \neq S$ o Let $\mathcal{C}$ denote the collection of all maximal convex subsets of $S$ and, for any fixed $k \geq 1$, let $\pi=\left\{A_{1} \cup \cdots \cup A_{k}: A_{1}, \ldots, A_{k}\right.$ distinct members of $\left.\mathcal{C}\right\}$. Then $\pi \neq \varnothing$ and $\cap \pi=\operatorname{ker} S$.

If $\mathcal{C}$ is the collection of all maximal convex subsets of some set $S$, it is easy to show that $\cap \mathcal{C}=$ ker $S$. This paper provides an interesting and perhaps surprising analogue of this well-known result. Throughout the paper, conv $S$, int $S$, and ker $S$ will be used to denote the convex hull, interior, and kernel, respectively, for the set $S$.

Further, we will make use of these familiar definitions: For points $x, y$ in a set $S$, we say $x$ sees $y$ via $S$ if and only if the corresponding segment $[x, y]$ lies in $S$. A subset $T$ of $S$ is said to be a visually independent subset of $S$ if and only if for every $x, y$ in $T, x \neq y, x$ does not see $y$ via $S$.

Received by the editors April 1, 1974 and, in revised form, June 7, 1974. AMS (MOS) subject classifications (1970). Primary 52A05.

Key words and phrases. Convex kernel, maximal convex subsets, unions of convex sets. 
Theorem 1. Let $S$ be a closed subset of some linear topological space such that int $\operatorname{ker} S \neq \varnothing$ and $\operatorname{ker} S \neq S$. Let $\mathcal{C}$ denote the collection of all maximal convex subsets of $S$ and, for any fixed $k \geq 1$, let $\mathbb{M}=\left\{A_{1} \cup \cdots \cup\right.$

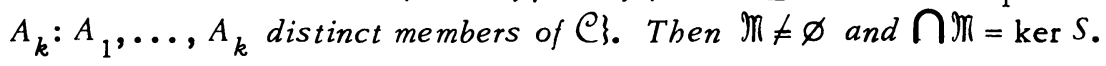

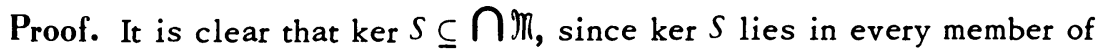
C. To prove the reverse inclusion, we show that if $x \in S$ and $x \notin \operatorname{ker} S$, there are infinitely many distinct members of $\mathcal{C}$ which fail to contain $x$. Since $x \notin \operatorname{ker} S$, we may select $p$ in $S$ with $[p, x] \nsubseteq \subseteq$. Also, select $z$ in int ker $S \neq \varnothing$. Clearly $z, p, x$ are not collinear. Because $S$ is closed, $[p, z] \subseteq S$ and $[p, x] \subseteq S$, there is some point $w$ on $[z, x)$ such that $p$ sees $w$ via $S$ and $p$ sees no point of $(w, x]$ via $S$. Also, since $z \in$ int $\operatorname{ker} S, w$ lies in the open interval $(z, x)$, and $\operatorname{conv}\{p, z, w\} \subseteq S$. Similarly, there is a point $y$ on $(z, p)$ such that $x$ sees $y$ via $S, x$ sees no point of $(y, p]$ via $S$, and conv $\{x, z, y\} \subseteq S$. Let $q$ denote the point of intersection of $(p, w)$ with $(x, y)$. There are two cases to consider.

Case 1. Assume for the moment that no point of $[p, q)$ sees any point of $[x, q)$ via $S$. Consider the family of segments $[a, b]$ supporting $\operatorname{conv}\{p, q, x\}$ at $q$, with $a$ on $[p, y)$ and $b$ on $[w, x)$. Each of these segments lies in a maximal convex subset of $S$ not containing $x$, and no two segments lie in the same maximal convex subset. Hence there are infinitely many maximal convex subsets of $S$ not containing $x$, and $x \notin \cap M$, the desired result.

Case 2. If some point of $[p, q)$ sees some point of $[x, q)$ via $S$, select points $p_{2}$ and $x_{2}$ having this property, with $p_{2}$ on $[p, q)$ and $x_{2}$ on $[x, q)$. Clearly $p_{2} \neq p$ and $x_{2} \neq x$, and we may select $p_{2}, x_{2}$ so that no point of $\left[p, p_{2}\right)$ sees any point of $\left[x, x_{2}\right)$ via $S$. Repeat an earlier argument to find points $w_{2}$ on $\left[x_{2}, q\right), y_{2}$ on $\left[p_{2}, q\right)$ such that $p_{2}$ sees $w_{2}$ via $S$ and $p_{2}$ sees no point of $\left(w_{2}, x\right]$ via $S, x_{2}$ sees $y_{2}$ via $S$ and $x_{2}$ sees no point of $\left(y_{2}, p\right]$ via $S$.

Without loss of generality, we assume that $p_{2} \neq y_{2}$ (for otherwise the following argument may be suitably adapted using $p, p_{2}, x_{2}$ in place of $p_{2}$, $q_{2}, x_{2}$, respectively). Let $q_{2}$ denote the point of intersection of $\left[p_{2}, w_{2}\right]$ with $\left[x_{2}, y_{2}\right]$. It is clear that $x$ sees no point on $\left[p_{2}, q_{2}\right] \cup\left(x_{2}, q_{2}\right]$. In case no point of $\left[p_{2}, q_{2}\right)$ sees any point of $\left[x_{2}, q_{2}\right)$ via $S$, we may repeat the argument of Case 1 to obtain an infinite collection of segments supporting conv $\left\{p_{2}, q_{2}, x_{2}\right\}$ at $q_{2}$, each of which lies in a maximal convex subset of $S$ not containing $x$, and no two of which lie in the same maximal convex subset of $S$, finishing the proof. 
Otherwise, some point of $\left[p_{2}, q_{2}\right)$ sees some point of $\left[x_{2}, q_{2}\right)$ via $S$, and we repeat the previous argument to obtain points $p_{3}, x_{3}, q_{3}$. Furthermore, $x$ cannot see $x_{3}$ via $S$. Continuing inductively, if for some $n$, no point of $\left[p_{n}, q_{n}\right)$ sees any point of $\left[x_{n}, q_{n}\right)$ via $S$, then the argument of Case 1 yields the desired result. If no such $n$ exists, then the infinite set of points $\left\{x_{2 n+1}: n \geq 1\right\}$ is a visually independent subset of $S$, no point of which sees $x$ via $S$. To each point $x_{2 n+1}$ we may associate a distinct maximal convex subset of $S$ not containing $x$. Therefore, $x \notin \cap M$. This completes Case 2 and the proof of the Theorem.

To see that the full hypothesis of Theorem 1 is required, consider the following example.

Example. For $k \geq 2$, let $x_{1}, \ldots, x_{k}$ denote $k$ distinct points of some line $L$, with $x_{1}<x_{2}<\cdots<x_{k}$, and let $y$ be a point not on $L$. If $S=$ int(conv $\left.\left\{x_{1}, x_{k}, y\right\}\right) \cup\left\{x_{1}, \ldots, x_{k}\right\}$, then $S$ is not closed, $S$ has exactly $k$ maximal convex subsets, and the corresponding set $\bigcap M$ is all of $S$.

Similarly, if $S$ is any collection of $k \geq 2$ distinct lines intersecting in a common point, then int $(\operatorname{ker} S)=\varnothing, S$ has exactly $k$ maximal convex subsets, and $\cap M=S$.

DEPARTMENT OF MATHEMATICS, UNIVERSITY OF OKLAHOMA, NORMAN, OKLAHOMA 73069 